\title{
ABSTRACTS
}

\section{INTERNATIONAL SYMPOSIUM ON}

HUMAN PURINE AND PYRIMIDINE METABOLISM

San Diego, California U.S.A.

July 28-August 1, 1985

ORGANIZING COMMITTEE

J. E. Seegmiller, Chairman

G. R. Bartlett

G. R. Boss

T. Friedmann

H. E. Gruber

S. S. Matsumoto

W. L. Nyhan

T. M. Page

L. F. Thompson

R. C. Willis 
THE EFFECT OF PHOSPHONOFORMIC ACID ON WILD TYPE AND

MUTANT S49 CELI LINES. D.A. Albert and L.J. Gudas.

Univ. of Chicago and Harvard Medical School.

Phosphonoformic acid (PFA) and its congener phosphonoacetic acid (PAN) are inhibitors of viral (and to a less extent mammalian) DNA polymerase. We selected mutants of 549 cells (a mouse $T$ lymphoma line) resistant to $3 \mathrm{mM}$ phosphonoformic acid. These li lines had a range of growth rates, cell cycle distribution abnor malities, and concomitant resistance to the inhibitory effects of thymidine, acycloguanosine (acyclovir), aphidicolin, deoxyadenosine, and novobiocin: Most lines had normal to sliqhtly elevated pools of ribonucleoside triphosphates and deoxyribonucleoside triphosphates. However, one line (PFA 3-9) had a greatly elevated dCTP pool and increased CDP reducatase activity in permeabilized cells. This activity in the PFA 3-9 cells diminished to wild type control levels in the presence of phosphonoformic acid, while PFA greatly diminished wild type CDP reductase activity (see table). In addition, 4mM PFA diminished the dCTP pool in wild type and in PFA 3-9 cells at 8 hours. The reduced dCTP pool could be increased by exogenous deoxycytidine but this only marginally reversed PFA toxicity. These observations suggest that PFA is an inhibitor of mammalian ribonucleotide reductase and partial resistance to PFA can be effected by mutation to increased CDP reductase activity resulting in a large dCTP pool.

Growth CDP Reductase dCIP dTrP dATP dOTP : Control Activity

wild Type

$+4 \mathrm{mM}$ PFA

PFA 3.9

$$
100 \quad 4855
$$

4855

$11.4 \quad 1605$

30.846 .0

$+4 \mathrm{mM}$ PFA $(8 \mathrm{hr}$ $\begin{array}{llll}30.8 & 46.0 & 31.9 & 14.0 \\ 14.4 & 26.6 & 34.9 & 12.7\end{array}$

$\begin{array}{rrrr}137.8 & 59.9 & 37.5 & 20.1 \\ 70.0 & 26.6 & 34.9 & 12.7\end{array}$

CHARACTERIZATION OF HYDROXYUREA(HYU) RESISTANT S49 $T$

2 LYMPHOMA CELLS. D. Albert and L.J. Gudas. Univ. Of

Chicago and Harvard Medical School.

We selected hydroxyurea resistant $549 \mathrm{~T}$ lymphoma cells by exposing wild type cells to increasing concentrations of hydroxyureaan inhibitor of ribonucleotide reductase that binds to the M2 subunit of the enzyme. These 10 to 60 fold resistant cell lines demonstrated increased ribonucleotide reductase activity and deoxyribonucleoside triphosphate pools consistent with the hypothesis that the activity of the M2 subunit of ribonucleotide reductase is rate limiting for both ribonucleotide reductase activity and deoxyribonucleoside triphosphate production. Assay of partially purified cell extracts containing M1 or M2 demonstrated the increased M2 activity in HYU resistant cells. Two dimensional gel electrophoresis demonstrated a single spot of increased in tensity ( $\mathrm{PI}=5.5 \mathrm{M}$.W. $50 \mathrm{~K}$ daltons) in HYU resistant cells which was proportional to the increased M2 activity. In addition hydroxyurea resistant cells were partially resistant to deoxyadenosine toxicity. Cytoflourimetry of HYU resistant cells showed a normal cell cycle distribution which was unchanged after exposure to dibutyryl cyclic AMP in contrast to wild type 549 cells which accumulate in Gl $(>90 \%)$. Similarly hydroxyurea resistant cells did not arrest in Gl when exposed to Forskolin as wild type S49 cells do. However, both hydroxyurea resistant and wild type cells were arrested in Gl phase with the phosphodiesterase inhibitor R0-1724. These data suggest that hydroxyurea resistant cells have increased ribonucleo -tide reductase activity due to increased M2 activity, possibly secondary to amplified genes(s) and exhibit abnormalities in cyclic AMP response.

PURINE DE NOVO SYNTHESIS AND SALVAGE DURING TESTICULAR DEVELOPMENT IN THE RAT

3 Jennifer Allsop and Richard W.E. Watts

Division of Inherited Metabolic Diseases

MRC Clinical Research Centre, Harrow HA1 3UJ, U.K.

Purine de novo synthesis and salvage occur in the testis. Cell division accelerates at puberty. We have correlated changes in the activity of purine de novo synthesis and purine salvage with the histology of the developing testis and with serum hormone levels. Purine de novo synthesis peaked at 17 days and HPRT activity at 35 days. APRT activity decreased at 17 days and remained very low. The 17 day peak of purine de novo synthesis coincided exactly with the appearance of spermatids and prominent spermatocytic meiosis. The 35 day HPR'i peak coincided with the first appearance of active spermatogenesis, full thickness of precursor cells and fully fledged spermatozoa. These findings indicate that the full development. of reproductive function in the male requires HPRT as well is purine de novo synthesis.

ALLOSTERIC CONTROL OF CTP SYNTHETASE IN VIVO.

4 Bruce Aronow and Buddy UIIman. Univ. Of $\frac{\mathrm{KY}}{\mathrm{K}}$

MedicaT Center, Dept. of Biochemistry, Lexington, KY, U.S.A. It has long been appreciated that CTP synthetase is an allosteric enzyme that is inhibited by its endproduct, CTP. The isolation and characterization of mutant murine $T$ lymphblasts with a genetically altered CTP synthetase activity which is refractory to inhibition by CTP have allowed an in situ determination of the essential regulatory factors governing pyrimidine synthesis and pyrimdine pool balance in a mammalian cell line. Metabolic flux and nucleotide pool measurements in unperturbed cycling cells and in cells in which the cellular pools have been exogenously manipulated indicated that the regulation of flux through CTP synthetase in situ occurs mainly by allosteric inhibition by CTP. Metabolic flux through CTP synthetase was arrested by imperceptible perturbations in CTP pools in wildtype cells but not in the mutant cells. Small changes in the levels of either ATP or GTP, a substrate and activator of the enzyme, respectively, had little influence on the in situ activity of CTP synthetase. fashion by uridylate nucleotides in wildtype but not in mutant cells. Thus, uridylate nucleotides govern the rates of de novo pyrimidine synthesis, while CTP modulates the balance between pyrimidine synthesis, while CTP modul
uridylate and cytidylate nucleotides.

5 ROLE OF THE NUCLEOSIDE TRANSPORT FUNCTION IN THE TRANSPORT, INCORPORATION, AND SALVAGE OF

HYPOXANTHINE. Bruce Aronow and Buddy UI lman. Univ. KY Medical Center, Dept. of Biochemistry, Lexington, KY, U.S.A. Genetic deficienceis in the nucleoside transport function markedly altered the abilities of cultured mutant $549 \mathrm{~T}$ lymphoblasts to transport, incorporate, and salvage exogenous hypoxanthine. The concentrations of hypoxanthine required to reverse azaserine toxicity and replenish azaserine-depleted nucleoside triphosphate pools in $A E_{1}$ cells, a nucleoside transport-deficient clone, were about 10-fold higher than those required for wildtype cells. Surprisingly, a second nucleoside transport-deficient clone, 80-502, which had lost $80-90 \%$ of its ability to transport nucleosides, required lower hypoxanthine concentrations to reverse these azaserine-mediated effects. The addition of 10uM p-nitrobenzylthioinosine (NBMPR), a potent inhibitor of nucleoside transport, to wildtype cells mimicked the phenotype of the $A E_{1}$ cells with respect to the

hypoxanthine reversal of the effects of azaserine. $\mathrm{AE}_{1}$ cells or NBMPR-treated wildtype cells could only transport

hypoxanthine at $20 \%$ the rate of untreated wildtype cells The 80-5-D2 cell line could transport and utilize hypoxanthine more efficiently than the wildtype parental cell line. A genetic deficiency in nucleoside transport did not interfere with adenine transport, incorporation, or salvage, however. These studies on $\$ 49 T$ iymphoblasts altered in their nucleoside transport capacity provide powerful genetic evidence that hypoxanthine and nucleosides share a common transport function.

ALTERATION OF RIBONUCLEOTIDE AND DEOXYRIBONUCLEOTIDE

6 METABOLISM BY INTERFERON IN HUNAN B-LYMPHOBLASTOID CELLS. Jerzy Barankiewicz, Chaim Kaplinsky and Amos Cohen. Division of Immunology, Research Instit

The effect of interferon on nucleic acid synthesis and on nucleotide metabolism was studied in human B-lymphoblastoid cells. Cells were incubated with 50 units/ml of recombinant $\alpha$-interferon for $24 \mathrm{hrs}$. Synthesis of nucleic acids, ribonucleotides, deoxyribonucleotides as we11 as nucleotide interconversion and catabolism were determined. Interferon inhibited both DNA and RNA synthesis measured by the incorporation of radioactive thymidine and uridine respectively. Ribonucleotide and deoxyribonucleotide biosynthesis by both the de novo and salvage pathways was markedly inhibited by interferon whereas nucleotide catabolism was significantly increased. Interferon also decreased intracellular PRPP levels but had no effect on ribonucleotide interconversion or protein synthesis measured by incorporation of radioactive threonine, leucine and valine. These results showed that inhibition of nucleic acid synthesis by interferon is associated with decreased availability of nucleotides for synthesis of both DNA and RNA. 